\title{
Diseño y validez de la Escala de Adicción a Instagram de Bergen (BIAS) en adultos peruanos
}

\section{Design and validity of the Bergen Instagram Addiction Scale (BIAS) in Peruvian adults}

\author{
Alexandra Chavez Santamaria
}

Universidad Nacional Federico Villarreal, Perú

ORCID: https://orcid.org/0000-0003-4350-1561

Miguel Angel Vallejos-Flores*

Universidad Nacional Federico Villarreal, Perú Asociación Peruana de Profesionales de las Adicciones, Perú

ORCID: https://orcid.org/0000-0002-6380-3412

*Correspondence

Email: mvallejosf@unfv.edu.pe
Cite as:

Chavez, A., \& Vallejos-Flores, M. (2021). Diseño y validez de la Escala de Adicción a Instagram de Bergen (BIAS) en adultos peruanos. Propósitos y Representaciones, 9(1), e973. Doi: http://dx.doi.org/10.20511/pyr2021.v9n1.973 
Resumen

Las redes sociales virtuales han ejercido un impacto notable en nuestras vidas, y con el pasar de los años se ha dado lugar a la creación de nuevas plataformas de socialización virtual como Instagram, la cual en nuestro contexto ha tomado gran interés, sobre todo en adultos jóvenes, llegando a ocupar gran parte de su espacio personal, académico, laboral y social, conduciendo en muchas ocasiones a comportamientos problemáticos como la adicción. El objetivo de este estudio fue diseñar y validar la Escala de adicción a Instagram de Bergen (BIAS), basada en la versión breve al español del Bergen Facebook Addiction Scale (BFAS). Participaron 200 adultos peruanos usuarios de Instagram de 18 a 35 años $\left(\mathrm{M}_{\text {edad }}=25.9\right.$; $\left.\mathrm{DE}_{\text {edad }}=4.29\right)$ el $63.5 \%$ fueron mujeres. Para el diseño se realizó el cambio de la terminología Facebook por Instagram, en los ítems del BFAS, posteriormente se efectuó el AFC para estimar las evidencias de validez factorial y AFC-MG para la invarianza de la medición por sexo, comprobando invariabilidad para todos los niveles (configural, métrica, fuerte y estricta). Se aplicaron los instrumentos; Escala de Bienestar Psicológico (BIEPS-A) y Escala de Satisfacción con la vida (SWLS) los cuales fueron relacionados con el BIAS corroborando la divergencia entre los constructos, asimismo, se analizó la convergencia del BIAS al ser correlacionado con el BFAS $(\mathrm{r}=.520)$. La fiabilidad del instrumento se estimó con los coeficientes Alfa $(\alpha=.925)$ y Omega $(\omega=.931)$. Se concluye que el BIAS posee óptimas evidencias de validez y fiabilidad, demostrando una estructura unidimensional para la medición de adicción a Instagram.

Palabras clave: Adicción, Instagram, Invarianza Factorial, Validez, Fiabilidad.

\section{Summary}

Virtual social networks have had a notable impact on our lives, and over the years it has led to the creation of new virtual socialization platforms such as Instagram, which in our context has taken great interest, especially in adults young people, occupying a large part of their personal, academic, work and social space, often leading to problematic behaviors such as addiction. The objective of this study was to design and validate the Bergen Instagram Addiction Scale (BIAS), based on the short Spanish version of the Bergen Facebook Addiction Scale (BFAS). 200 Peruvian adult Instagram users from 18 to 35 years old participated $(\mathrm{M}$ age $=25.9$; $\mathrm{SD}$ age $=$ 4.29), 63.5\% were women. For the design, the terminology was changed from Facebook to Instagram, in the items of the BFAS, later the AFC was carried out to estimate the evidence of factorial validity and AFC-MG for the invariance of the measurement by sex, checking invariance for all the levels (configural, metric, strong and strict). The instruments were applied; the Psychological Well-being Scale (BIEPS-A) and the Life Satisfaction Scale (SWLS), which were related to the BIAS, corroborating the divergence between the constructs, and the convergence of the BIAS was analyzed as it was correlated with the BFAS $(\mathrm{r}=.520)$. The reliability of the instrument was estimated with the Alpha $(\alpha=.925)$ and Omega $(\omega=.931)$ coefficients. It is concluded that the BIAS has optimal evidence of validity and reliability, demonstrating a onedimensional structure for measuring addiction to Instagram.

Keywords: Addiction, Instagram, Factorial Invariance, Validity, Reliability.

\section{Introducción}

En este contexto de crisis mundial por la pandemia las redes sociales virtuales se han convertido en una herramienta fundamental para amortiguar el impacto de la pandemia y sostener la crisis socioeconómica a la que población mundial se enfrentaba. El gran despliegue de los medios virtuales facilitó la socialización y sobrellevar el aislamiento social, pues trajeron consigo importantes beneficios como, posibilitar un contacto comunicacional inmediato, brindar información, oportunidades para afianzar trabajos y emprendimiento en línea, entretenimiento y más. Lo expuesto, permite apreciar múltiples aspectos positivos que trae consigo el uso de novedosos medios sociales virtuales, pues se enfocan en satisfacer necesidades básicas de 
comunicación y socialización para el ser humano, fundamento que, podría explicar el gran avance y la popularidad que han alcanzado (Araujo, 2016; Colás et al., 2013).

Sin embargo, es importante mencionar que muchos de las redes sociales virtuales, que poseen auge a nivel mundial, han vislumbrado acciones inadecuadas en el hombre, tal es el caso de; la pérdida en el control frente a su uso, y el desarrollo de consecuencias negativas que a pesar de su aparición, no han frenado el uso excesivo hacia estos medios, llegando en muchos casos a ejercer un comportamiento adictivo, en tanto, según informa Andreassen (2015) los criterios en mención son fundamentales al hablar de adicciones comportamentales, frente a medios como las redes sociales virtuales.

Entorno a lo detallado, se puede inferir, que si bien las redes sociales virtuales, benefician y son necesarios para el hombre en sus diversos ámbitos de desarrollo, es importante conocer y diferenciar lo que implica un uso adecuado de los mismos, frente al desarrollo de comportamientos problemáticos, pues recientes estudios certifican que el ejercer un uso inadecuado de las tecnologías y de las redes sociales virtuales, suele relacionarse con la aparición de problemas como, cyberloafing, ciberadicción, nomofobia, phubbing, miedo a perderse algo o Síndrome de FOMO, entre otros (García del Castillo, 2013; Mejía, 2014; Arnaiz et al., 2016; Capilla \& Cubo, 2017; Coskun \& Gokcearslan, 2019; Varchetta et al., 2020; Metin-Orta \& Demirutku, 2020).

Según la información estadística reportada por Emarsys (2019) en el mundo, existe un aproximado de 3200 millones de personas que hacen uso de redes sociales, estos datos reflejan que el $40 \%$ de la población en el mundo accede a dichos medios, siendo Facebook, la red social de mayor uso a nivel mundial (El Sahili, 2015) con un aproximado de 2500 millones de usuarios que se encuentran activos de forma mensual (Statista, 2020) y es sobre dicha plataforma que surgen actualmente numerosos trabajos de investigación, enfocados en el desarrollo de elementos evaluativos, que facilitan la medición del comportamiento adictivo frente a dicha plataforma como; el Bergen Facebook Addiction Scale (BFAS; Andreassen et al., 2012) una herramienta que adquiere óptimas medidas de validez y fiabilidad, ejecutadas inicialmente en Europa y Asia, pero que cuenta con revisiones en Latinoamérica y en el Perú, las cuales refirman que es una herramienta útil para la medición de la adicción a Facebook en nuestro contexto y que además se muestra como un instrumento breve y conciso, de amplia contribución a la psicología de las adicciones (Vallejos-Flores, Copez-Lonzoy y Capa-Luque, 2018). No obstante, existen otras plataformas virtuales de reciente aparición que han adquirido una importante aceptación a nivel social, tal es el caso de; Instagram, definida como una red social de rápido desarrollo y fácil acceso (Rojas \& Panal, 2017).

Si bien, Instagram, a nivel mundial tan solo se encuentra dentro del ranking de las cinco redes sociales de mayor uso, esta plataforma es la que ha tenido mayor crecimiento, pues ya en el 2020 mantiene un total de 1000 millones de usuarios de forma mensual, a tan solo diez años de su creación (Statista, 2020). Dicha red social se ha instaurado como la plataforma virtual, de mayor proyección a nivel mundial y local, siendo considerada en muchos contextos, como un campo de interés para evaluar el desarrollo de comportamientos adictivos, tal es así que, en Asia y Europa existen instrumentos como; The Instagram Addiction Scale (TIAS; Sholeh \& Rusdi, 2019) procedente de Indonesia, Instagram Addiction Scale (IAS; Kircaburun \& Griffiths, 2018) en Turquía, Test for Instagram Addiction (TIA; D’Souza et al., 2018) en India, entre otros.

De acuerdo a lo referido, existen investigadores que han puesto énfasis en el desarrollo de herramientas enfocadas en la evaluación de conductas problemáticas y adictivas sobre plataformas virtuales como Instagram, sin embargo, estos estudios vienen desarrollándose con mayor predominio en contextos como Europa y Asia, dato sumamente importante, pues si bien la información estadística de Statista (2020) reporta que en dichos sectores el acceso a este medio es muy frecuente, pues, en países como India, existen al mes por lo menos 80 millones de usuarios de Instagram, en Indonesia unos 63 millones de usuarios, en Turquía unos 38 millones, cuyos 
grupos de mayor acceso a nivel mundial suelen tener entre 18 a 44 años, representando al $81.5 \%$ de usuarios sobre dicha red social (Gotor, 2019; Statista, 2020; We are social \& Hootsuite, 2020).

Pese a ello, sectores como Latinoamérica, no son la excepción, por el contrario, un informe del diario El Peruano (2020) reveló que Instagram es la segunda red social de mayor uso en dicho territorio, es decir, por lo menos un $80 \%$ de los latinos emplean dicho medio. Por consiguiente, Perú muestra datos estadísticos interesantes entorno al uso de Instagram reportando que un total de 7.1 millones de peruanos emplean dicha red social (Ipsos, 2020).

En síntesis, se muestra un evidente interés y un comprobado incremento en el acceso a medios de socialización virtual como Instagram, el cual, de no adquirir un uso adecuado, posibilita el desarrollo de comportamientos de uso problemático y el riesgo de desarrollar adicción sino se detectan tempranamente, ya que, pone en manifiesto la presencia de síntomas como; saliencia, que indicaría la relevancia, importancia y el dominio que ejerce el comportamiento adictivo en el sujeto. Síntomas de abstinencia, que muestra estados emocionales y respuestas físicas en el sujeto tras suspender su acceso al medio adictivo, de igual forma, las recaídas, junto con la variabilidad en el estado anímico, así como la tolerancia, que se entiende como el incremento en la actividad adictiva con el propósito de manifestar los efectos anteriormente producidos y finalmente, los conflictos acontecidos a causa del uso inadecuado a dichos medios (Andreassen, 2015; Griffiths \& Szabo, 2014; Kircaburun \& Griffiths, 2018).

Como bien, se expresó en párrafos anteriores, las personas de mayor accesibilidad a Instagram suelen tener edades entre los 18 a 44 años (Statista, 2020; We are social \& Hootsuite, 2020) considerados como un importante sector productivo en el campo académico laboral y por ende, de gran preocupación ya que una de sus principales herramientas en el estudio o trabajo son las tecnologías y las redes sociales virtuales, por lo que su uso inadecuado podría acarrearles problemas vinculados a estas, por ello, el desarrollar estudios vinculados al análisis de comportamientos problemáticos y adictivos son de suma importancia para evitar problemas de mayor envergadura asociados a las adicciones como problemas de aprendizaje, baja productividad académica, desmotivación (Sharma, 2020) conflictos laborales, baja producción, accidentes laborales, estrés laboral, entre otros (Aghaz \& Sheikh, 2016; Andel et al., 2019).

Asimismo, las estadísticas en nuestro contexto hacen referencia que por lo menos un $39 \%$ de usuarios de Instagram, suelen dedicar de 1 a 3 horas al día a dicha plataforma, mientras que, el $10.6 \%$ dedica un poco más de 3 horas diarias, además, de forma mensual el promedio de peruanos pasa unas 25 horas en esta red social, así mismo, los estudios señalan que, los peruanos navegamos en Instagram el doble de tiempo que el promedio de latinos (Comscore, 2018; Vizcarra, 2019).

Estas evidencias invitan a plantear investigaciones en nuestro contexto, referente a dicho tema, sin embargo, surge la interrogante de: ¿cómo evaluar el comportamiento adictivo a Instagram? si en la actualidad no se cuentan con herramientas de cribado en nuestro medio que permitan tal análisis, por ello, es necesario diseñar y validar siguiendo los lineamientos de la metodología y diseño instrumental (Ato et al., 2013) que facilite la obtención de medidas válidas y fiables para nuestro ámbito y que se aporte con este novedoso y necesario tema, considerando como base para este estudio con propuestas previas y adaptadas ya a nuestro medio como, la versión breve al español de la Bergen Facebook Addiction Scale (BFAS; Andreassen et al., 2012) que es un instrumento de rápida aplicación y utilidad, que puede incluirse en protocolos evaluativos, como herramienta de apoyo junto con otras técnicas para los profesionales de la salud, para su valoración del uso adictivo a Facebook, instrumento que ha demostrado propiedades psicométricas aceptables como lo reportaron Vallejos-Flores et al. (2018) en universitarios, informando que el BFAS presenta un muy buen ajuste sobre dicho constructo, pero que, a su vez está direccionado tan solo hacia la red social Facebook, no obstante, sus indicadores responden propiamente a criterios vinculados a adicciones comportamentales, las cuales pueden adaptarse a otras redes sociales como Instagram, pues es también una red social, y posee funciones similares a Facebook, motivo por el cual este trabajo plantea por objetivo; diseñar y hallar las propiedades psicométricas de la Escala de Adicción a Instagram de Bergen (BIAS), basada en la 
versión breve al español del BFAS (Balcerowska et al., 2020) y así consignar evidencias de validez y fiabilidad sobre dicho instrumento en adultos peruanos, de igual forma, resulta importante generar aportes sobre la invariabilidad del instrumento, en función a elementos como el sexo, debido a que, existen precedentes de que al evaluar comportamientos similares a la adicción a Instagram, como es el caso de las adicciones hacia sustancias, ludopatía, entre otros, suelen presentarse algunas diferencias en varones y mujeres (Blanco et al., 2005). En ese sentido, datos en específico sobre redes sociales, informan que los varones suelen tener mayor atracción a actividades que impliquen entretenimiento, mientras que, las mujeres buscan primordialmente construir relaciones sociales, apreciándose intereses distintos frente a las redes sociales virtuales en varones y mujeres (Chan et al., 2015). De igual forma, consignando los datos del portal Statista (2020) en adultos de 18 a 34 años, se manifiesta mayor presencia de usuarios de Instagram varones, que mujeres, reportando una acogida de mayor aceptabilidad en dicho grupo. En síntesis, es por ello, que este estudio, además de analizar las evidencias de validez y confiabilidad, pretende evaluar la invarianza de la medición de la Escala de adicción a Instagram de Bergen (BIAS) de acuerdo al sexo, justificando aportes relevantes en materia psicométrica y en líneas de investigación como la psicología de las adicciones.

\section{Método}

\section{Participantes}

La muestra de la investigación estuvo constituida por 200 adultos peruanos usuarios de la red social Instagram de 18 a 35 años, quienes fueron seleccionados a través de un muestreo por conveniencia (Otzen \& Manterola, 2017) es decir, se incluyeron los casos donde los sujetos contaban con accesibilidad al estudio. Para determinar el tamaño de la muestra se aplicaron criterios para modelos SEM (Ventura-León et al., 2020) consignando datos en la calculadora de tamaño muestral Soper (2020) tomando en cuenta el tamaño anticipado de efecto, con un valor mínimo de 0.30 para modelos factoriales, potencia estadística de 0.95 , probabilidad a un nivel 0.05 , una variable latente y seis variables observadas (estructura de la prueba), obteniendo como recomendación considerar un mínimo de 200 casos para el análisis efectivo de los datos. El $63.5 \%$ de los participantes fueron mujeres y un $36.5 \%$ varones. La media de edad de los participantes fue de 25.9 ( $\mathrm{DE}$ edad $=4.29)$. La totalidad de los participantes refiere emplear la red social Instagram, mientras que, un $69 \%$ señala un uso frecuente de dicha red social, por otro lado, el $50.5 \%$ de los evaluados refirió cursar estudios universitarios, mientras que, un $52.5 \%$ se encontraba laborando.

\section{Instrumentos}

Escala de Adicción a Instagram de Bergen (BIAS) es una versión modificada del Bergen Facebook Addiction Scale (BFAS; Andreassen et al., 2012) y la versión breve en español, conformada por 6 reactivos, escala validada por Vallejos-Flores et al. (2018) en el contexto peruano. La composición final de los ítems se realizó reemplazando la palabra Facebook del BFAS, por el término Instagram, tomando en consideración que ambos medios son espacios de socialización virtual y responden a criterios de adicciones comportamentales (Andreassen, 2015; Griffiths \& Szabo, 2014; Kircaburun \& Griffiths, 2018; Balcerowska et al 2020). En ese sentido, la prueba cuenta con seis ítems, de estructura unidimensional. Además, presenta una escala Likert en sus opciones de respuesta $(1=$ muy raramente, $2=$ raramente, $3=$ a veces, $4=$ a menudo, $5=$ muy a menudo) y permite obtener un puntaje total del constructo adicción a Instagram, sumando el global de respuestas halladas en los seis ítems que conforman la escala.

Bergen Facebook Addiction Scale (BFAS) instrumento diseñado por Andreassen et al. (2012) para la medición de la adicción a Facebook. La versión breve y en español fue validada en el contexto peruano por Vallejos-Flores et al (2018). Esta versión breve se encuentra constituido por seis ítems, posee también un escalamiento tipo Likert en sus respuestas (muy raramente a muy a menudo). La validez de constructo del instrumento reportó en un análisis factorial confirmatorio que se ajusta a una estructura unidimensional (CFI=.994, TLI=.990, SRMR=.032, 
RMSEA=.061), finalmente en la confiabilidad muestra un coeficiente adecuado $(\alpha=.84)$ (Vallejos-Flores et al., 2018).

Escala de Bienestar Psicológico (BIEPS-A) desarrollada por Casullo (2002) en Argentina, es un instrumento dirigido a personas de 18 a más años, cuenta con 13 ítems, que responden a 4 dimensiones, con tres opciones de respuesta $(1=$ en desacuerdo, $2=$ ni de acuerdo ni en desacuerdo, $3=$ de acuerdo). En Lima, Domínguez (2014) reportó la validez del instrumento a través de un análisis factorial confirmatorio, el cual presentó un ajuste adecuado (CFI=.961; $\mathrm{GFI}=.895 ; \mathrm{AGFI}=.839$; $\mathrm{RMR}=.032$; $\mathrm{RMSEA}=.091)$ que corroboró la adecuación de los cuatro componentes iniciales en la prueba. Respecto a la confiabilidad, el análisis de consistencia interna señaló un valor general en el estadístico Alfa de Cronbach de .966; y en sus componentes coeficientes oscilantes desde .836 a .908 .

Escala de Satisfacción con la vida (SWLS) instrumento elaborado por Diener et al. (1985) cuenta con 5 ítems, y está dirigido para población adulta. Posee estructura unidimensional, y opciones de respuesta bajo un escalamiento tipo Likert $(1=$ totalmente en desacuerdo, $2=$ en desacuerdo, $3=$ indiferente, $4=$ de acuerdo, $5=$ totalmente de acuerdo). Calderón et al. (2018) en contexto peruano reportó la validez de constructo del instrumento, refiriendo los siguientes coeficientes $(\mathrm{CFI}=.998 ; \mathrm{RMSEA}=.052 ; \mathrm{SRMR}=.027)$ confirmando un ajuste adecuado en el modelo unidimensional de la prueba, en cuanto a la confiabilidad señalaron un valor adecuado de $\omega=.90$

\section{Procedimiento}

Como primer paso se procedió a realizar el cambio de la terminología Facebook por Instagram, en los seis ítems que conforman el Bergen Facebook Addiction Scale (BFAS) versión breve al español (Vallejos-Flores et al., 2018). Se diseñó un cuestionario online desarrollado mediante la herramienta Google Formularios compuesto por una ficha sociodemográfica, el consentimiento informado y las escalas anteriormente referidas, considerando un tiempo aproximado de evaluación de 15 minutos. En el cuestionario los participantes fueron informados sobre los objetivos de la investigación, de la forma de participación de manera anónima y voluntaria, así como del manejo confidencial de los datos y de la importancia del cumplimiento de los principios de la bioética en investigación; autonomía, de justicia, no maleficencia y beneficencia (Amaro et al., 1996). Los participantes aceptaron las condiciones del estudio en la página inicial del formulario antes de responder a las escalas. Finalmente, se analizaron los datos para dar cumplimiento a los objetivos del estudio y discutir los hallazgos.

\section{Análisis de datos}

El análisis de los datos se aplicó bajo la estadística descriptiva e inferencial. Para el análisis de los ítems se aplicaron los estadísticos; media (M) desviación estándar (DE), coeficiente de asimetría de Fisher (g1), coeficiente de curtosis de Fisher (g2), comunalidad (h2) e índice de homogeneidad corregida (IHC). En cuanto a las evidencias de validez basada en la estructura interna, se realizó el análisis factorial confirmatorio (AFC) haciendo uso de matrices policóricas, dado que, los datos responden a un escalamiento ordinal, el método empleado fue el Mínimo Cuadrados Ponderados con medias y varianzas ponderadas (WLSMV) (Brown, 2015). Los valores referentes para considerar un ajuste adecuado en el modelo se tomaron en base a lo reportado por Escobedo et al. (2015) tal es el caso de; medida de parsimonia $\left(\mathrm{X}^{2} / \mathrm{gl}\right) \leq 3.00$, la raíz del error cuadrático media de aproximación (RMSEA) $\leq 0.80$, índice de ajuste comparativo $(\mathrm{CFI})>.90$, índice Tucker Lewis (TLI) $>.90$ y la raíz promedio de residuos estandarizados $(\mathrm{SRMR}) \leq .08$. Respecto a las evidencias de validez basada en la relación con otros constructos (convergente y divergente), se emplearon las medidas de correlación de Pearson (r) y el tamaño del efecto $\left(\mathrm{r}^{2}\right)$ (Cohen, 1988) con el propósito de correlacionar el constructo adicción a Instagram con adicción a Facebook, bienestar psicológico y satisfacción con la vida. De igual forma, se realizó el análisis de equivalencia de medición, considerando la variable sexo. Para ello, se aplicó las medidas de ajuste al Análisis Factorial Confirmatorio (AFC-MG) pero a nivel multigrupos, 
llegando a analizar los diversos grados de invarianza (configural, métrica, fuerte y estricta) (Pendergast et al., 2017) evaluando las diferencias en el índice de ajuste comparativo (CFI), tomando $(\triangle \mathrm{CFI}<0,01)$ como valor adecuado (Cheung \& Rensvold, 2002). Por último, se analizó la confiabilidad de la escala a través del método de consistencia interna con los estadísticos Alfa de Cronbach $(\alpha)$ y Omega de McDonald's ( $\omega$ ) (Cascaes et al., 2015; Cho, 2016). Los análisis de datos, fueron efectuados con el programa R Studio 3.5.1 y el programa Jamovi 1.1.9.0.

\section{Resultados}

\section{Análisis de ítems}

En la tabla 1 se aprecia la descripción de ítems que componen la escala BIAS, obteniendo su puntuación media (M) la cual fue menor para el reactivo 4 "¿utilizó Instagram tanto que tuvo un impacto negativo en su trabajo o estudios?", asimismo, se dan a conocer los valores de la desviación estándar (DE) los cuales fueron menores al puntaje de la media. En cuanto al IHC los coeficientes oscilaron entre (.81 a .89) situándose óptimos, pues fueron superiores a 30 (Kline, 2010). La varianza común compartida (h2) se situó por encima de .70. Los valores obtenidos en la asimetría (g1) fueron aceptables en todos los reactivos, dado que, se hallaron menores a 3. En cuanto a la curtosis (g2) se obtuvieron valores entre (-.92 a 4.89) considerándose adecuados, puesto que, Kline (2010) señala presencia de problemas cuando dicho indicador no se ubica en el rango $-10 \mathrm{a}+10$.

\section{Tabla 1}

Análisis descriptivo de ítems de la Escala de Adicción a Instagram de Bergen (BIAS)

\begin{tabular}{cccccccc}
\hline Ítem & $\mathrm{M}$ & $\mathrm{DE}$ & $\mathrm{g} 1$ & $\mathrm{~g} 2$ & $\mathrm{~h} 2$ & $\mathrm{IHC}$ & Aceptable \\
\hline 1 & 1.87 & .979 & .589 & -.928 & .740 & .801 & $\mathrm{Si}$ \\
2 & 1.41 & .765 & 2.01 & 3.86 & .909 & .894 & $\mathrm{Si}$ \\
3 & 1.65 & 1.007 & 1.44 & 1.26 & .847 & .885 & $\mathrm{Si}$ \\
4 & 1.35 & .720 & 2.22 & 4.89 & .800 & .825 & $\mathrm{Si}$ \\
5 & 1.48 & .814 & 1.78 & 2.80 & .856 & .881 & $\mathrm{Si}$ \\
6 & 1.52 & .874 & 1.69 & 2.36 & .828 & .873 & $\mathrm{Si}$ \\
\hline
\end{tabular}

Nota. Media (M), desviación estándar (DE), coeficiente de asimetría de Fisher (g1), coeficiente de curtosis de Fisher (g2), comunalidad (h2) e índice de homogeneidad corregida (IHC).

\section{Evidencias de validez basada en la estructura interna}

Se aprecia que los pesos factoriales en la escala BIAS fueron robustos $(\lambda>.80)$, adicionalmente, los valores de los IC 95\% se ubicaron entre .793 a .983 (Tabla 2). Asimismo, en la tabla 3, considerando que los reactivos responden a un escalamiento ordinal, podemos observar la matriz de correlaciones policóricas de los ítems del instrumento, cuyos coeficientes se situaron entre .74 a .89. En cuanto al análisis factorial confirmatorio, se empleó con la finalidad de corroborar la estructura factorial unidimensional del instrumento, obteniendo un ajuste adecuado en dicho modelo $\left(\mathrm{X}^{2} / \mathrm{gl}=2.13\right.$; RMSEA $=.075 ; \mathrm{CFI}=.998 ; \mathrm{TLI}=$.996; $\left.\mathrm{SRMR}=.021\right)$ (Escobedo et al., 2015) (Tabla 4). 


\section{Tabla 2}

Pesos factoriales en la Escala de Adicción a Instagram de Bergen (BIAS)

\begin{tabular}{lllll}
\hline $\begin{array}{l}\text { Ítems } \\
\text { ¿Con qué frecuencia durante el último año usted ha. ..? }\end{array}$ & $\mathrm{P} \lambda$ & \multicolumn{2}{c}{$\lambda$ IC 95\% } \\
$\begin{array}{l}\text { 1. ¿pasado buen tiempo pensando en Instagram o } \\
\text { planeando su uso? }\end{array}$ & .865 & $(.793$ & $.922)$ \\
$\begin{array}{l}\text { 2. ¿sentido inquietud o preocupación cuando se le } \\
\text { prohibió el uso de Instagram? }\end{array}$ & .955 & $(.904$ & $.983)$ \\
$\begin{array}{l}\text { 3. ¿usado Instagram con el fin de olvidar problemas } \\
\text { personales? }\end{array}$ & .920 & $(.868$ & $.953)$ \\
$\begin{array}{l}\text { 4. ¿utilizado Instagram tanto que tuvo un impacto } \\
\text { negativo en su trabajo o estudios? }\end{array}$ & .902 & $(.820$ & $.940)$ \\
$\begin{array}{l}\text { 5. ¿tratado de reducir el uso de Instagram sin éxito? } \\
\text { 6. ¿sentido el impulso de usar Instagram cada vez más? }\end{array}$ & .923 & $(.860$ & $.962)$ \\
\hline
\end{tabular}

Nota. Pesos factoriales (P $\lambda)$

\section{Tabla 3}

Correlaciones policóricas entre los ítems de la Escala de Adicción a Instagram de Bergen (BIAS)

\begin{tabular}{ccccccc}
\hline Ítem & 1 & 2 & 3 & 4 & 5 & 6 \\
\hline 1 & - & & & & & \\
2 & 0.86 & - & & & & \\
3 & 0.79 & 0.86 & - & & & \\
4 & 0.74 & 0.83 & 0.88 & - & - & \\
5 & 0.79 & 0.89 & 0.84 & 0.84 & - & \\
6 & 0.82 & 0.89 & 0.81 & 0.79 & 0.84 & - \\
\hline
\end{tabular}

Nota. Ítem 1: saliencia; 2: síndrome de abstinencia; 3: modificación en el estado de ánimo; 4: conflictos; 5: recaídas; 6: tolerancia

\section{Tabla 4}

Ajuste al Análisis Factorial Confirmatorio de la Escala de Adicción a Instagram de Bergen (BIAS)

\begin{tabular}{cccccc}
\hline Modelo & X $^{2} / \mathrm{gl}$ & RMSEA & CFI & TLI & SRMR \\
\hline BIAS 6 & 2.13 & .075 & .998 & .996 & .021 \\
\hline
\end{tabular}

Nota. $X^{2} / g l=$ medida de parsimonia; RMSEA= raíz del error cuadrático media de aproximación; $\mathrm{CFI}=$ índice de ajuste comparativo; $\mathrm{TLI}=$ índice Tucker Lewis; $\mathrm{SRMR}=$ raíz promedio de residuos estandarizados. 


\section{Invarianza de la medición según sexo}

Se estableció la invarianza de medida, en función a la variable sexo, el valor que se consignó a evaluar fue la diferencia del CFI $(\Delta \mathrm{CFI})$ considerando que sea menor a .01 (Cheung \& Rensvold, 2002) para admitir la invariabilidad del constructo en los grupos analizados. A pesar de imponer las restricciones, se llegó a consignar que el constructo presenta invariabilidad estricta $(\triangle \mathrm{CFI}=$ .00) en función al sexo, indicando que no se presenta deterioro del modelo al ser evaluado el constructo en grupos diferenciados por sexo (Tabla 5).

\section{Tabla 5}

Análisis de invarianza de la medición en la Escala de Adicción a Instagram de Bergen (BIAS), según sexo

\begin{tabular}{cccccccc}
\hline Sexo & CFI & TLI & RMSEA & $\mathrm{X}^{2} / \mathrm{gl}$ & $\Delta$ CFI & $\Delta$ TLI & $\begin{array}{c}\Delta \text { RMSE } \\
\mathrm{A}\end{array}$ \\
\hline Mujeres & 0.983 & 0.968 & 0.068 & 2.02 & - & - & - \\
Varones & 0.991 & 0.983 & 0.080 & 1.51 & - & - & - \\
Configura & 0.996 & 0.992 & 0.037 & 1.76 & - & - & - \\
l & & & & & & - \\
Métrica & 0.990 & 0.986 & 0.067 & 1.44 & 0.004 & 0.008 & 0.046 \\
Fuerte & 0.999 & 0.999 & 0.011 & 1.39 & 0.000 & 0.000 & 0.013 \\
Estricta & 0.981 & 0.982 & 0.075 & 1.56 & 0.000 & 0.002 & 0.013 \\
\hline
\end{tabular}

Nota. CFI= índice de ajuste comparativo; TLI= índice Tucker Lewis; RMSEA= raíz del error cuadrático media de aproximación; $\mathrm{X}^{2} / \mathrm{gl}=$ medida de parsimonia; $\Delta \mathrm{CFI}=$ diferencias de CFI; $\Delta$ $\mathrm{TLI}=$ diferencias de TLI; $\Delta \mathrm{RMSEA}=$ diferencias de RMSEA.

\section{Evidencias de validez basada en la relación con otros constructos}

Se presenta el resultado de la correlación de Pearson (r) entre la escala BIAS, que mide el uso adictivo a Instagram, con un constructo similar, tal es el caso del uso adictivo a Facebook consignado a través de la escala BFAS, obteniendo una correlación positiva $(\mathrm{r}=.520)$ con un tamaño de efecto pequeño $\left(\mathrm{r}^{2}=.270\right)$ situándose en el rango de .100 a .300 (Cohen, 1988) mientras que, al correlacionar el instrumento BIAS, con otros constructos totalmente distintos como es el caso del BIEPS-A que evalúa bienestar psicológico y SWLS que evalúa satisfacción con la vida, el resultado señaló un efecto nulo o ausencia de efecto pues $\left(\mathrm{r}^{2}\right)$ fue menor a .100 (Cohen, 1988; Tornimbeni, 2004; Meneses et al., 2013) (Tabla 6).

\section{Tabla 6}

Correlaciones entre el constructo adicción a Instagram, con la adicción a Facebook, bienestar psicológico y satisfacción con la vida

\begin{tabular}{ccccc}
\hline & & BFAS & BIEPS-A & SWLS \\
\hline \multirow{3}{*}{ BIAS } & $\mathrm{R}$ & $.520^{* *}$ & $-.216^{* *}$ & $-.180^{*}$ \\
& $\mathrm{P}$ & .000 & .002 & .011 \\
& $\mathrm{r}^{2}$ & .270 & .046 & .032 \\
\hline
\end{tabular}

Nota. Correlación de Pearson (r), significancia estadística $(p)<.05$, tamaño del efecto $\left(r^{2}\right)$. 


\section{Fiabilidad}

Se comprueba la fiabilidad del instrumento a través del método de consistencia interna, exponiendo una excelente fiabilidad pues los valores obtenidos fueron superiores a .90, tanto al ser efectuados con el estadístico Alfa de Cronbach's $(\alpha=.925)$ como también con el estadístico Omega de McDonald's $(\omega=.931)$ (George \& Mallery, 2003).

\section{Discusión}

Se propuso contribuir con una herramienta de apoyo al diagnóstico temprano de los comportamientos adictivos, en la línea de las adicciones tecnológicas, específicamente a las adicciones a redes sociales, que están emergiendo como nuevos trastornos comportamentales que preocupa a la comunidad científica ( Días, et al. 2018; Vallejos-Flores et al. 2018; Andel et al 2019; Coskun y Gokcearslan, 2019; Balcerowska et al 2020; Orta y Demirutku, 2020; Sharma, 2020) siendo Instagram una de estas redes sociales con mayor crecimiento en los últimos, amenazando con quitar la preferencia a la red social Facebook y adquiriendo gran relevancia en los adultos jóvenes (Kircaburun \& Griffiths, 2018) debido a ello, resulta importante contribuir con herramientas de medición para la detección temprana del uso problemático o adictivo, tal y como se ha venido realizando en otros países (D’Souza et al., 2018; Sholeh \& Rusdi, 2019).

En ese contexto, se indagaron medidas validadas en nuestro País, que respalden teóricamente una versión inicial del instrumento, seleccionando el Bergen Facebook Addiction Scale (BFAS) en su versión breve reportada por Vallejos-Flores et al. (2018) como un instrumento de medida que ha reportado evidencias de validez para el constructo adicción a Facebook, dicho instrumento fundamenta una estructura unidimensional y responde a los criterios para las adicciones comportamentales, evaluando los indicadores de saliencia, síndrome de abstinencia, modificación en el estado de ánimo, conflictos, recaídas y tolerancia (Griffiths, 2005; Andreassen, 2015) los cuales, se ajustan de forma muy similar al hablar de uso adictivo a Instagram, pues en ambas redes sociales virtuales, encontramos semejanzas en sus estrategias de uso y contenido, siendo el tiempo de creación a favor de Facebook, lo que le da mayor popularidad a dicha red social, no obstante, Instagram pese a tener menor tiempo de aparición en internet, ha generado notoriedad a nivel mundial y en nuestro contexto, es por ello, que se comprobó a través del análisis factorial confirmatorio (AFC) si el modelo empleado para el uso adictivo a Facebook, cumplía también con las evidencias de validez para el uso adictivo de la red social Instagram, hallándose según los análisis un ajuste adecuado y comprobándose la unidimensionalidad de la Escala de Adicción a Instagram de Bergen (BIAS), adicionalmente, se pudo evidenciar que los pesos factoriales en la escala BIAS, fueron similares que en el BFAS, al igual que el índice de ajuste comparativo (CFI) e índice Tucker Lewis (TLI), igualmente el valor de la raíz promedio de residuos estandarizados (SRMR) y de la raíz del error cuadrático media de aproximación (RMSEA) fueron próximos a los reportados para el BFAS (Vallejos-Flores et al., 2018; Días et al., 2018) demostrándose de esta manera que el BIAS, presenta índices de ajuste adecuados evidenciando validez de estructura interna.

En la misma línea explicativa sobre los indicadores de la adicción a Instagram, Sholeh y Rusdi (2019) construyeron una escala de medida de la adicción a Instagram siguiendo los seis indicadores que se usan para evaluar las adicciones comportamentales, indicadores que se consignaron en el presente trabajo, no obstante, dichos autores consideraron a los indicadores como dimensiones y realizaron los estudios psicométricos reportando que su instrumento presentaba evidencias de validez aceptables, con pesos factoriales adecuados, pero menores a los obtenidos en esta investigación. De la misma manera, al comparar nuestros hallazgos obtenidos con otros instrumentos de medida de la adicción a Instagram por ejemplo con el de Kircaburun y Griffiths (2018) quienes también se basaron en los mismos criterios de adicción comportamental, reportaron la existencia de dos factores, y que su modelo presentaba un ajuste adecuado y con 
pesos factoriales óptimos que oscilaban desde .54 a .77 , índices por debajo de lo hallado en este estudio (.74 a .89). Si bien los estudios reportados son de otras latitudes, no obstante, son citados para respaldar nuestros hallazgos, debido a la ausencia de estudios psicométricos de este constructo, por tanto, podemos señalar que el BIAS presenta mejores evidencias psicométricas comparados con otros estudios reportados en otros contextos.

De igual forma, buscando garantizar las evidencias de validez del BIAS también se realizó análisis de validez en relación con otras variables, haciendo uso de un constructo similar, tal es el caso del uso adictivo a Facebook consignado a través de la escala BFAS, obteniendo una correlación positiva, con un tamaño de efecto pequeño, estos resultados confirmaron que la adicción a Instagram se encuentra relacionada directamente con la adicción a Facebook, demostrándose evidencias de convergencia de la escala y corroborando que está midiendo lo que pretende medir, tomando en cuenta que el BFAS, es un instrumento que ampliamente ha evidenciado evaluar adicción a Facebook, por lo que el hecho de evidenciar relación con el BIAS, demuestra que este último también mide adicción a las redes sociales, de manera específica al Instagram (Andreassen, 2015; Vallejos-Flores et al., 2018; Días et al., 2018). Del mismo modo, se realizó la evaluación del BIAS, con otros instrumentos que evalúan diferentes constructos como el BIEPS-A que evalúa bienestar psicológico y SWLS que evalúa satisfacción con la vida, que al relacionarlos con el BIAS se evidenció ausencia de efecto en la correlación, con un coeficiente de correlación pobre con tendencia a negativo, evidencias que indican que la prueba BIAS, mide un constructo totalmente opuesto a las que miden las escalas BIEPS-A y SWLS, ya que, la adicción es una variable incompatible a la presencia de bienestar psicológico o satisfacción vital (Burga et al., 2015). De esta forma se puntualiza evidencias de validez de la medida (Días et al., 2018).

Por otro lado, en este estudio se propuso indagar la invarianza factorial del constructo adicción a Instagram, respecto al sexo, observándose que no se presentaba deterioro del modelo al ser evaluado en grupos diferenciados por sexo, por lo que podemos señalar que la estructura factorial es invariante según el sexo. Nuestros hallazgos que se asemejan a lo reportado por otros autores sobre la invarianza factorial de instrumentos que evalúan adicciones a redes sociales y adicción a Facebook (Vallejos-Flores et al., 2018; Salas et al., 2020). A las luces de las evidencias halladas respecto a la invarianza de medición podemos señalar que la Escala de Adicción a Instagram de Bergen (BIAS) no presenta sesgos asociados al sexo. (Dominguez-Lara y PradaChapoñan, 2019; Caycho-Rodriguez et al., 2020)

Del mismo modo, se logró corroborar la fiabilidad del instrumento asumiendo coeficientes de consistencia interna que lo sitúan en un nivel excelente para dicha medida (George \& Mallery, 2003) hallazgos similares a los reportados por Salas et al. (2020) quienes informaron valores superiores a .90. Así también, al ser comparada la fiabilidad del BIAS, con estudios del ámbito internacional que investigaron propiedades psicométricas de la adicción a Instagram, nuestros hallazgos reflejan coincidencias (D’Souza et al., 2018; Sholeh \& Rusdi, 2019).

Finalmente, podemos señalar que el BIAS presenta adecuadas propiedades psicométricas convirtiéndose en un instrumento de utilidad para la evaluación temprana de indicadores de adicción al Instagram. A pesar de ser conscientes de las limitaciones que podría asumirse debido al tamaño de la muestra, no obstante, una muestra mayor posibilitaría otros análisis y con mejores alcances estadísticos, más aun, para la ejecución de análisis de invarianza factorial con otros criterios. Pese a ello, los análisis realizados brindaron índices de ajustes aceptables que aseveran la presencia de un modelo unidimensional adecuado y con propiedades psicométricas adecuadas, sin embargo, resulta conveniente, que los interesados en replicar el estudio, empleen una muestra de mayor tamaño, dando lugar a posibilitar análisis de invariabilidad con otros elementos sociodemográficos, como también se sugieren estudios destinados a valorar los puntos de corte del instrumento para identificar casos de adicción. 


\section{Referencias}

Aghaz, A. y Sheikh, A. (2016). Cyberloafing and job burnout: An investigation in the knowledge intensive sector. Computers in Human Behavior, 62, $51-60$. https://doi.org/10.1016/j.chb.2016.03.069

Amaro, M., Marrero, A., Valencia, M., Casas, S. y Moynelo, H. (1996). Principios básicos de la bioética. Revista cubana de enfermería, 12(1). http://scielo.sld.cu/scielo.php?script=sci_arttext\&pid=S0864-03191996000100006

Andel, S., Kessler, S., Pindek, S., Kleinman, G., y Spector, P. (2019). Is cyberloafing more complex than we originally thought? Cyberloafing as a coping response to workplace aggression exposure. Computers in Human Behavior, 101, 124-130. https://doi.org/10.1016/j.chb.2019.07.013

Andreassen, C. (2015). Online social network site addiction: A comprehensive review. Current Addiction Reports, 2(2), 175-184. http://dx.doi.org/10.1007/s40429-015-0056-9

Andreassen, C., Torsheim, T., Brunborg, G., \& Pallesen, S. (2012). Development of a Facebook addiction scale. Psychological Reports, 110(2), 501-517. http://dx.doi.org/10.2466/02.09.18.PR0.110.2.501-517

Araujo, E. (2016). Indicadores de adicción a las redes sociales en universitarios de Lima. Revista Digital de Investigación en Docencia Universitaria, 10(2). http://dx.doi.org/10.19083/ridu.10.494

Arnaiz, P., Cerezo, F., Giménez, A. y Maquillón, J. (2016). Conductas de ciberadicción y experiencias de cyberbullying entre adolescentes. Anales de Psicología, 32(3), 761-769. http://dx.doi.org/10.6018/analesps.32.3.217461

Ato, M., López, J. y Benavente, A. (2013). Un sistema de clasificación de diseños de investigación en psicología. Anales de psicología, 29(3), 1038-1059. http://redalyc.org/articulo.oa?id=16728244043

Balcerowska, J., Bereznowski, P., Biernatowska, A., Atroszko, P., Pallesen, S. y Andreassen, C (2020). Is it meaningful to distinguish between Facebook addiction and social networking sites addiction? Psychometric análisis of Facebook addiction and social networking sites addiction scales. Current Psychology. https://doi.org/10.1007/s12144-020-00625-3

Blanco, P., Sirvent, C. y Palacios, L. (2005). Diferencias de género en la adicción e implicaciones terapéuticas. Salud y drogas, 5(2), 81-97. http://www.redalyc.org/articulo.oa?id=83905205

Brown, T. (2015). Confirmatory factor analysis for applied research (2da. Ed.). Guilford

Burga, J., Fernández, M., Llanca, L., Tafur, D., Vera, P. y Díaz, C. (2015). Nivel de satisfacción con la vida personal y riesgo de adicción a Facebook en estudiantes de instituciones educativas en una región del Perú. Revista Hispanoamericana De Ciencias De La Salud, 1(2), 87-93. http://www.uhsalud.com/index.php/revhispano/article/view/55

Capilla, E. y Cubo, S. (2017). Phubbing. Conectados a la red y desconectados de la realidad. Un análisis en relación al bienestar psicológico. Pixel-Bit. Revista de Medios y Educación, 50, 173-185. http://www.redalyc.org/articulo.oa? $\mathrm{id}=36849882012$

Cascaes, F., Gonçalves, E., Valdivia, B., Bento, G., Da Silva, T., Soleman, S. y Da Silva, R. (2015). Estimadores de consistencia interna en las investigaciones en salud: el uso del coeficiente alfa. Revista peruana de medicina experimental Salud Pública, 32(1), 129-138. https://www.scielosp.org/article/rpmesp/2015.v32n1/129-138/es/

Casullo, M. (2002). Evaluación del Bienestar Psicológico en Iberoamérica. Paidós.

Caycho-Rodríguez T., Ventura-León, J., Azabache-Alvarado, K., Reyes-Bossio, M. y CabreraOrosco, I. (2020). Validez e invariancia factorial del Índice de Bienestar General (who-5 wbi) en universitarios peruanos. Revista de Ciencias de la Salud, 18(3), 1-23. https://revistas.urosario.edu.co/index.php/revsalud/article/view/9797

Chan, T., Cheung, C., Shi, N. y Lee, M. (2015). Gender differences in satisfaction with Facebook users. Industrial Management \& Data Systems, 115(1), 182-206. Doi: 10.1108/IMDS-082014-0234 
Cheung, G. y Rensvold, R. (2002). Evaluating goodness-of-fit indexes for testing measurement invariance. Structural Equation Modeling: A Multidisciplinary Journal, 9(2), 233-255. Doi: 10.1207/s15328007sem0902_5

Cho, E. (2016). Making Reliability Reliable. Organizaonal Research Methods, 19(4), 651-682. hps://doi.org/10.1177/1094428116656239

Cohen, J. (1988). Statistical Power Analysis for the Behavioral Sciences. Erlbaum, Hillsdale.

Colás, P., González, T. y De Pablos, J. (2013). Juventud y redes sociales: Motivaciones y usos preferentes. Comunicar, 20(40), 15-23. http://dx.doi.org/10.3916/C40-2013-02-01

Comscore. (13 de Abril de 2018). Estado de Social Media en América Latina 2018. Comscore. https://www.comscore.com/lat/Prensa-y-Eventos/Presentaciones-y-librosblancos/2018/Estado-de-Social-Media-en-America-Latina-2018

Coskun, T. y Gokcearslan, S. (2019). Examination of cyberloafing studies in education: A content analysis. $\quad$ World Journal on Educational Technology: Current Issues, $\quad 11(1), \quad 94-$ 103. https://doi.org/10.18844/wjet.v11i1.4017

Dias, P., Cadime, I., del Castillo-López, A., García-Castillo, F. y García del Castillo, J. (2018). Excessive facebook use among portuguese university students: contributes of the adaptation of the Bergen facebook addiction. Salud $y$ drogas, 18(1), 131-139. https://www.researchgate.net/publication/323218156_Excessive_facebook_use_among_ Portuguese_university_students_Contributes_of_the_adaptation_of_the_bergen_facebo ok_addiction

Dominguez, S. (2014). Análisis psicométrico de la escala de bienestar psicológico para adultos en estudiantes universitarios de Lima: un enfoque de ecuaciones estructurales. Psychologia: avances de la disciplina, $8(1), \quad 23-31$. http://www.scielo.org.co/pdf/psych/v8n1/v8n1a03.pdf

Dominguez-Lara, S. y Prada-Chapoñan, R. (2020). Invarianza de medición y datos normativos de una medida breve de inteligencia emocional en estudiantes universitarios peruanos. Anuario de Psicología, 50(2), 87-97. https://revistes.ub.edu/index.php/Anuariopsicologia/article/view/31932

D’Souza, L., Samyukta, A. y Tevin, J. (2018). Development and Validation of Test for Instagram Addiction (TIA). The International Journal of Indian Psychology, 6(3), 4-14. Doi: $10.25215 / 0603.81$

El Peruano. (16 de Marzo de 2020). Facebook es la red social más popular en el Perú. El Peruano.https://elperuano.pe/noticia-facebook-es-red-social-mas-popular-el-peru93094.aspx

El Sahili, L. (2015). Psicología de facebook: Vislumbrando los fenómenos psíquicos, complejidad y alcance de la red social más grande del mundo. Trillas.

Emarsys. (3 de Enero de 2019). Top 5 Social Media Predictions for 2019. Emarsys. https://emarsys.com/learn/blog/top-5-social-media-predictions-2019/

Escobedo, M., Hernández, J., Estebané, V. y Martínez, G. (2015). Modelos de Ecuaciones Estructurales: Características, Fases, Construcción, Aplicación y Resultados. Ciencia y trabajo, 18(55), 16-22. https://scielo.conicyt.cl/pdf/cyt/v18n55/art04.pdf

Garcia del Castillo, J. (2013). Technological addictions: the rise of social networks. Salud $y$ Drogas, 13 (1), 5-14. Doi: 10.21134/haaj.v13i1.188

George, D. y Mallery, P. (2003). Spss for Windows step by step: A Simple Guide and Reference. (4ta. Ed.). Allyn \& Bacon.

Gotor, D. (31 de Julio de 2019). Informe e-País en Turquía. Icex. https://www.icex.es/icex/wcm/idc/groups/public/documents/documento/mdiw/odqw/ edi sp/doc2020840605.pdf?utm_source=RSS=ICEX.es=17-01-2020=Informe\%20e$\mathrm{Pa} \% \mathrm{C} 3 \%$ ADs\%20Turqu\%C3\%ADa\%202019

Griffiths, M. (2005). A component model of addiction within a biopsychosocial framework. Journal of Substance Use, 10(4). Doi:10.1080/14659890500114359

Griffiths, M. y Szabo, A. (2014). Is excessive online usage a function of medium or activity? An empirical pilot study. Journal of Behavioral Addictions, 3, 74-77. Doi: 10.1556/JBA.2.2013.016 
Ipsos. (18 de Marzo de 2020). Redes sociales en el Perú Urbano. Ipsos. https://www.ipsos.com/espe/redes-sociales-en-el-peru-urbano

Kline, R. (2010). Principles and practice of structural equation modeling (3ra Ed). Guilford Press.

Kircaburun, K. \& Griffiths, M. (2018). Instagram addiction and the Big Five of personality: The mediating role of self-liking. Journal of Behavioral Addictions, 7(1), 158-170. https://doi.org/10.1556/2006.7.2018.15

Lorenzo-Seva, U., Timmerman, M. E., \& Kiers, H. (2011). The Hull method for selecting the number of common factors. Multivariate Behavioral Research, 46, 340-364. Doi: 10.1080/00273171.2011.564527

Mejía, G. (2014). Sexting: una modalidad cada vez más extendida de violencia sexual entre jóvenes. Perinatología y reproducción humana, 28(4), 217-221. http://www.scielo.org.mx/pdf/prh/v28n4/v28n4a7.pdf

Meneses, J., Barrios, M., Bonillo, A., Cosculluela, A., Lozano, L., Turbany, J. y Valero, S. (2013). Psicometría. Editorial UOC.

Metin-Orta, I. y Demirutku, K. (2020). Cyberloafing behaviors among university students and its relation to Hedonistic-Stimulation value orientation, cyberloafing attitudes, and time spent on the Internet. Current Psychology. https://doi.org/10.1007/s12144-020-00932-9

Otzen, T. y Manterola, C. (2017). Técnicas de muestreo sobre una población a estudio. International Journal of Morphology, 35(1), 227-232. https://scielo.conicyt.cl/pdf/ijmorphol/v35n1/art37.pdf

Pendergast, L., Von der Embse, N., Kilgus, S. \& Eklund, K. (2017). Measurement Equivalence: A Non-Technical Primer on Categorical Multi-Group Confirmatory Factor Analysis in School Psychology. Journal of School Psychology, 60, 65-82. Doi: 10.1016/j.jsp.2016.11.002

Rojas, J. y Panal, A. (2017). El uso de Instagram en los medios de comunicación deportivos. Análisis comparado de Bleacher Report, L'équipe y Marca. Ámbitos, 38, 1-20. http://www.redalyc.org/articulo.oa?id=16853353005

Salas, E., Copez, A., Merino, C. (2020). ¿Realmente es demasiado corto? Versión breve del cuestionario de adicción a redes sociales (ARS-6). Salud y drogas, 20(2), 105-118. Doi: 10.21134/haaj.v20i2.536

Sharma, S (2020) I want it my way: Using consumerism and neutralization theory to understand students' cyberslacking behavior. International Journal of Information Management, 53 (2020), 102-131. https://doi.org/10.1016/j.ijinfomgt.2020.102131

Sholeh, A. y Rusdi, A. (2019). A New Measurement of Instagram Addiction: Psychometric Properties of The Instagram Addiction Scale (TIAS). CISAK, 91-97. https://www.researchgate.net/publication/326656045_Developement_The_Instagram_Ad diction_Scale_TIAS

Soper, D. (2020). A-priori Calculadora de tamaño de muestra para modelos de ecuaciones estructurales 2020. Free Statistics Calculators. http:// www.danielsoper.com/statcalc

Statista. (27 de Enero de 2019). Number of daily active Instagram Stories users from October 2016 to January 2019 (in millions). Statista. https://www.statista.com/statistics/730315/instagram-stories-dau/

Statista. (27 de Junio de 2020). Ranking de las principales redes sociales a nivel mundial según el número de usuarios mensuales activos en enero de 2020. Statista. https://es.statista.com/estadisticas/600712/ranking-mundial-de-redes-sociales-pornumero-de-usuarios/

Statista. (24 de Marzo de 2020). Distribución porcentual de los usuarios de Instagram a nivel mundial en 2020, por género y edad. Statista. https://es.statista.com/estadisticas/875368/distribucion-por-genero-y-edad-de-losusuarios-mundiales-de-instagram/

Tornimbeni, S. (2004). Introducción a los tests psicológicos. Brujas.

Tresáncoras, A., García-Oliva, C. y Piqueras, J. (2017). Relación del uso problemático de Whatsapp con la personalidad y la ansiedad en adolescentes. Salud y drogas, 17(1), 27-36. https://ojs.haaj.org/index.php/haaj/article/view/272 
Vallejos-Flores, M., Copez-Lonzoy, A. y Capa-Luque, W. (2018). ¿Hay alguien en línea?: Validez y fiabilidad de la versión en español de la Bergen Facebook Addiction Scale (BFAS) en universitarios. Salud y drogas, 18(2), 175-184. https://ojs.haaj.org/index.php/haaj/article/view/394

Varchetta, M., Fraschetti, A., Emanuela, M. y Giannini, A. (2020). Adicción a redes sociales, Miedo a perderse experiencias (FOMO) y Vulnerabilidad en línea en estudiantes universitarios. Revista Digital de Investigación en Docencia Universitaria, 14(1), 1-13. http://dx.doi.org/10.19083/ridu.2020.1187

Ventura-León, J., Caycho, T. y Barboza, M. (2020). ¿Cuántos participantes son necesarios?: Un método para estimar el tamaño muestral en SEM. Archivos argentinos de pediatría, 118(5), 510- 513.

Vizcarra, E. (22 de junio de 2019). ¿Cuánto tiempo pasamos en redes sociales?. La República. https://larepublica.pe/tecnologia/2019/06/22/cuanto-tiempo-pasamos-en-redes-sociales/

We are social \& Hootsuite.(2020). Digital 2020 Global Digital Overview. Juancmejia https://www.juancmejia.com/wp-content/uploads/2020/02/Digital-2020-WeAreSocial-yHootSuite.pdf 